Iulia Ciolachi

DMD

Holistic Dental \& Medical Institute

of Bucharest-ROPOSTURO

Bucharest, Romania

e-mail:

dr.iuliaciolachi@yahoo.ro

\section{Management of \\ Dental Emergencies \\ in Children \\ and Adolescents}

Editors: Klaus W. Neuhaus, Adrian Lussi

Publisher:Wiley-Blackwell, Hoboken, NJ, USA

Language: English

ISBN: 978-1-119-37264-6

Edition: 1/e

Publish Year: 2019

Pages: 320 , illustrated

Price: $€ 67.80$

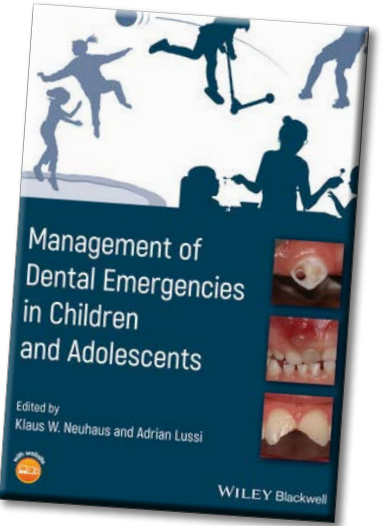

In order to provide effective and adequate dental care to children and adolescents, general practitioners, pedodontists and non-pediatric specialists must always be up-to-date with novel treatment protocols.

Dr. Klaus W. Neuhaus and Prof. Adrian Lussi present the first edition of the book entitled Management of Dental Emergencies in Children and Adolescents based on the Swiss school experience in pediatric dental emergencies.

This book is divided into seven units including twenty-four chapters.

Unit 1, General Considerations for Emergency Management in Children and Adolescents recapitulates the main features of tooth development and pulp biology, offers guidelines for the correct indication for antibiotic therapy, behavioural management besides some basic considerations of conscious sedation and management of radiographic needs.

Unit 2, Management of Tooth Substance Loss presents methods for managing the deep carious lesion, biological foundation, diagnostic approaches and appropriate treatment modalities for root-fractured teeth, crown and crown-root fractures.

Unit 3, Management of Open Pulp in Permanent Teeth describes the pulpotomy protocol after trauma, how to perform pulpectomy with open apex and a step-by-step treatment protocol reflecting regenerative endodontics.

Unit 4, Management of Open Pulp in Deciduous Teeth explains methods to carry out a full coronal pulpotomy, the clinical procedure and the outcome of pulpectomy and tooth extractions indication.

Unit 5 develops extensively aspects of Management of Missing Teeth such as resin-bonded restorations, treatment protocol of an avulsed permanent tooth, ankylosis and external root resorption after trauma and orthodontic treatment aspects at various ages.

Unit 6, Management of Oral Health Conditions, focuses on the most common viral diseases affecting the oral mucosa, with special emphasis on children, non-infective swellings and oral problems in patients undergoing haematology or oncology treatment.

Unit 7, Management of Non-infective Dental Conditions covers molar - incisor hypomineralisation (MIH) treatment, clinical management of dentin hypersensitivity (DH) and cracked tooth syndrome (CTS) treatment.

This book is a unique manual for general practitioners, non-pediatric specialists and pedodontists, accompanied by a companion website and has numerous images, graphs, radiographs and also clinical cases. This book will help you manage potentially stressful situations with children and adolescents while improving your competence in a wide range of urgent pediatric situations.

d) http://www.stomaeduj.com 10.25241/stomaeduj.2020.7(2).bookreview.7

The Books Review is drafted in the reviewer's sole wording and illustrates his opinions. 